\title{
EUROSTERONE MEETING
}

\section{AP-1-glucocorticoid receptor crosstalk taken to a higher level}

\author{
M Karin and L Chang \\ Laboratory of Gene Regulation and Signal Transduction, Department of Pharmacology, UCSD School of Medicine, 9500 Gilman Drive, La Jolla, \\ California 92093, USA \\ (Requests for offprints should be addressed to M Karin, Department of Pharmacology, University of California, San Diego, 9500 Gilman Drive, La Jolla, \\ California 92093-0636, USA; Email: karinoffice@ucsd.edu)
}

\begin{abstract}
More than a decade ago our view of gene regulation by glucocorticoids (GC) and other steroid hormones underwent a dramatic change with the discovery of negative crosstalk (transcriptional interference) between the GC receptor (GCR) and transcription factor AP-1 (Jun:Fos). It was initially observed that induction of the collagenase type 1 gene, which is mediated through activation of AP-1 by growth factors and inflammatory cytokines, is repressed by GC. This repression was attributed to mutual negative interactions between AP-1 and GCR. Although the exact molecular mechanism underlying this particular case of transcriptional interference is yet to be determined, it has become clear that this and analogous interactions with
\end{abstract}

other transcription factors (e.g. nuclear factor- $\kappa \mathrm{B}$ ) underlie the anti-inflammatory and immunosuppressive activity of GC. Recent studies conducted at the whole animal level indicate that the interactions between the AP-1 and GC signaling pathways are much more extensive. AP-1related signaling via the Jun $\mathrm{N}$-terminal kinases can lead to increased levels of circulating GC, which eventually down-modulate AP-1 activity via transcriptional interference. This negative feedback loop is likely to be of great importance for maintenance of homeostasis and regulation of stress responses, including acute and chronic inflammation.

Journal of Endocrinology (2001) 169, 447-451

\section{Introduction}

In the beginning (mid to late 80 s) we had a rather simplified and naive view of gene regulation by glucocorticoid (GC) hormones, growth factors and cytokines. By binding to their receptor (GCR) which happens to be a sequence-specific DNA-binding protein, GC lead to gene induction following the recognition of positively acting GC response elements (GREs) by ligand-bound GCR. The two prime examples for this type of regulation were the murine mammary tumor virus (MMTV) long terminal repeat and the human metallothionein $\mathrm{II}_{\mathrm{A}}\left(\mathrm{hMTII}_{\mathrm{A}}\right)$ gene (reviewed in Beato 1989). Shortly thereafter, AP-1 was identified as a sequencespecific transcription factor involved in the induction of $\mathrm{hMTII}_{\mathrm{A}}$ and collagenase type I genes by phobol esters, growth factors and pro-inflammatory cytokines (reviewed in Angel \& Karin 1991). The two signaling pathways that regulate the activity of these transcription factors were thought to happily coexist in the nucleus without any exchange or interaction, except for promoters, such as that of the $\mathrm{hMTII}_{\mathrm{A}}$ gene, that contain binding sites for both GCR and AP-1 (Karin et al. 1984). However, analysis of the human collagenase promoter soon led to disruption of this idyllic but simple picture. While potently induced by growth factors and cytokines, expression of collagenase and other members of its family of metalloproteinases is strongly repressed by GC (Jonat et al. 1990). This form of negative regulation is of great clinical importance because prolonged induction of collagenase by growth factors and inflammatory cytokines, such as interleukin (IL)-1, is responsible for much of the tissue damage that occurs in rheumatoid arthritis, whereas the inhibition of collagenase induction by GC underlies their utility as anti-arthritic drugs (Kelly \& Griffiths 1989). Other examples for similarly regulated genes are those that code for IL-2, a major growth factor for T cells and other cytokines, such as interferon $\gamma$ or IL-1, whose transcription is stimulated in response to activation of AP-1 and other transcription factors (nuclear factor (NF)- $\mathrm{KB}, \mathrm{NF}-\mathrm{AT}$, STATs) and is repressed by GC (Barnes \& Karin 1997). It is believed that the repression of such genes underlies the utility of GC as immunosuppressive and anti-inflammatory drugs (Barnes \& Karin 1997, Karin 1998).

\section{Mechanisms}

Analysis of the collagenase (Jonat et al. 1990) and IL-2 (Auphan et al. 1995) promoter/enhancer regions had 
revealed that the segments that are sufficient for mediating repression by GC do not contain GREs or any other binding site for GCR. Furthermore, in both cases repression is rapid and in the case of collagenase independent of induction of GCR target genes. A search for physical interactions between GCR and the Jun and Fos components of AP-1 revealed low-affinity interactions detectable by co-precipitation of overexpressed proteins (Diamond et al. 1990, Jonat et al. 1990, Schüle et al. 1990, Yang-Yen et al. 1990). Furthermore, mobility shift experiments using recombinant or cell-free translated proteins revealed mutual inhibition of DNA-binding activity upon mixing of GCR with AP-1 proteins (Diamond et al. 1990, Jonat et al. 1990, Schüle et al. 1990, Yang-Yen et al. 1990). These early results led to the suggestion that the basis for transcriptional interference in this case is formation of a physical complex between GCR and AP-1 (Jun:Fos or Jun:Jun dimers), in which either participant is unable to recognize its cognate DNA-binding site. This simple model was quickly challenged by genomic footprinting experiments, which suggested that AP-1 remains bound to the collagenase promoter under conditions of GCmediated repression (Konig et al. 1992). Such results led to the suggestion that the interaction between GCR and promoter-bound AP-1 prevents the latter from productive interaction with the transcriptional initiation machinery or an essential co-activator complex and vice versa (Saatcioglu et al. 1994). It was also suggested that GCR and AP-1 simply compete for a common co-activator complex containing CREB-binding protein (CBP) or p300 (Kamei et al. 1996). However, as the amounts of nuclear CBP/p300 seem to exceed those of AP-1 or GCR and $\mathrm{CBP} / \mathrm{p} 300$ is also a common target for many other sequence specific transactivators, such as NF- $\mathrm{\kappa B}$ or STATs, which do not transrepress AP-1 activity, it is unlikely that simple competition for a limiting amount of $\mathrm{CBP} / \mathrm{p} 300$ can explain the transrepression of AP-1 activity by GCR. With the advent of the chromatin immunoprecipitation procedure, which can be used to examine the interactions of specific transcription factors and co-activator complexes with particular target genes (Braunstein et al. 1993, Alberts et al. 1998), it would be extremely worthwhile to examine the mechanism of mutual transcriptional interference using this powerful technique. For instance, an interaction with the activated GCR may prevent AP-1 docked to the collagenase promoter from recruiting certain co-activator complexes. Alternatively, this interaction may induce AP-1 to recruit a corepressor complex instead of a co-activator. The latter recruitment may in fact be mediated by GCR with an unoccupied DNA-binding domain, as it is possible that in the absence of DNA binding GCR may have a higher affinity for co-repressors than co-activators. Indeed, there is ample experimental evidence that binding of nuclear receptors to DNA results in an allosteric change that alters their transcriptional properties (Lefstin \& Yamamoto 1998).
A completely different explanation for transcriptional interference had focused on the effect of GC and other ligands for nuclear receptors on the signaling pathways that regulate AP-1 activity (Caelles et al. 1997, Swantek et al. 1997, Rogatsky et al. 1998). It is well established that growth factors and cytokines stimulate AP-1 activity through activation of different members of the mitogenactivated protein kinase family, such as extracellular signalregulated kinase, p38 and Jun N-terminal kinase (JNK) (Karin 1995). Of these, the most critical for stimulation of AP-1 activity are members of the JNK group, whose activity is stimulated by growth factors and even more so by pro-inflammatory cytokines, such as IL-1 and tumor necrosis factor (TNF) (Karin 1995). Once activated, the JNKs phosphorylate and thereby enhance the activity of sequence specific transcription factors involved in the induction of fos and jun gene transcription, as well as enhance the transcriptional activity of both pre-existing and newly synthesized Jun proteins (Smeal et al. 1991, Kallunki et al. 1996).

It has been shown that GC and other steroid hormones as well as thyroid hormone can reduce JNK activity by approximately 50\% (Caelles et al. 1997). This effect was shown to be receptor mediated, but is unlikely to be exerted by direct interaction between the activated receptors, which are nuclear proteins, and the bulk of JNK, which remains outside the nucleus even after it is activated (Cavigelli et al. 1995). It is also not clear how a mere 50\% reduction in JNK activity can account for the almost complete repression of AP-1 target genes, such as collagenase, by GC. In agreement with these findings, the recent paper by Gonzalez et al. (2000) demonstrates that inhibition of JNK activity by GC is greater within the nucleus than that observed in the cytoplasm and that this cannot be accounted for by a physical association between GCR and JNK. Further studies are required to delineate the mechanisms by which the activity of JNK is inhibited by GC inside the nucleus.

\section{Physiological importance}

Regardless of the exact mechanism used, the ability of GC to repress AP-1 as well as NF- $\kappa B$ activity is of great physiological significance. In fact, we would argue that the most important function of GC and GCR is not necessarily the classical activation of GRE-containing genes, but the repression via transcriptional interference of AP-1 and $\mathrm{NF}-\mathrm{\kappa B}$ activities. It has been shown that injection of normal mice with modest amounts of either bacterial endotoxin (LPS) or the potent T cell-activating anti-CD3 antibody results in some distress but not in lethality. However, when the same non-lethal doses of LPS or anti-CD3 are injected into adrenalectomized mice, severe lethality ensues (Auphan et al. 1995). This lethality is most likely due to deregulated production of pro-inflammatory 


\title{
Emotional
}

\section{stress}

\section{infection}

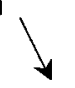

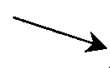
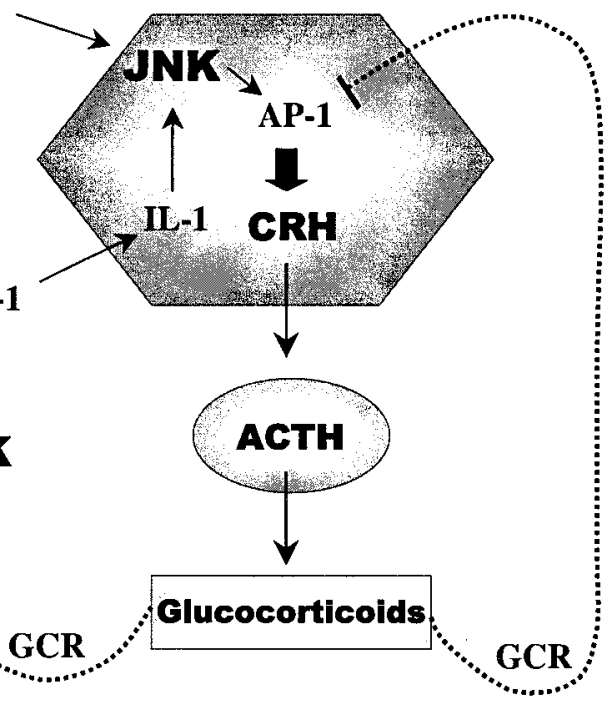

\section{Hypothalamus}

\author{
Pituitary
}

\section{Adrenal}

\section{inflammatory and tissue} remodeling genes

\begin{abstract}
Figure $1 \mathrm{JNK}$ is involved in activation of the HPA and plays both initiating and terminating roles in physiological responses to stress. Emotional stress or bacterial infection such as pro-inflammatory cytokine IL-1 activates JNK activity, resulting in elevation of AP-1 activity and CRH expression, which then induces the secretion of pituitary adrenocorticotropic hormone (ACTH) and, hence, acts on the adrenal cortex to cause the release of GCs. GCs are circulated to a variety of tissues where through binding to the GCRs they lead to inhibition of AP-1-mediated gene induction. Solid lines represent excitatory inputs, broken lines inhibitory ones.
\end{abstract}

cytokines, such as TNF and IL-1, caused by the massive and unopposed activation of NF- $\mathrm{KB}$ and AP-1 by LPS or anti-CD3.

More direct and better evidence for the importance of transcriptional interference or transrepression was recently provided by Schutz and his coworkers who generated a specific knock-in mutant mouse strain $G R^{\text {dim/dim }}$, whose GCR is unable to bind with high affinity to GRE due to defective dimerization but is still fully capable of repressing AP-1 and NF- $\kappa B$ activities (Reichardt et al. 1998). Interestingly, unlike $G R^{-1-}$ mice which die shortly after birth (Cole et al. 1995), GR ${ }^{\text {dim/dim }}$ mice appear relatively normal and healthy under standard laboratory conditions (Reichardt et al. 1998). As GR ${ }^{\mathrm{dim} / \mathrm{dim}}$ mice are unable to induce the expression of classical GCR target genes, such as tyrosine aminotransferase or MMTV, it appears that positive gene regulation by GC is not essential for survival, at least under laboratory conditions. However, it follows that negative transcriptional control via transcriptional interference, a function that is absent in $G R^{-1-}$ mice but present in $G R^{\text {dim/dim }}$ mice, is essential for survival even under controlled laboratory conditions. It is not clear, however, which genes need to be negatively regulated by GC to allow survival. As discussed previously, one essential function of GC that is likely to be mediated via transcriptional interference is the attenuation of cyclo-oxygenase 2 (COX2) expression (Karin 1998). Deregulated expression of COX2 may lead to severe alterations in blood pressure due to overproduction of prostaglandins and leukotienes.

\section{Crosstalk via the hypothalamic-pituitary-adrenal (HPA) axis}

Recently a new level of crosstalk between AP-1 and GCR was identified. The interactions in this case are largely indirect and are based on activation of the HPA axis. The HPA axis is the central neuroendocrine pathway responsible for maintaining homeostasis during stress. In response to stress, such as acute infection or emotional stress, neuronal input into the hypothalamus (mostly from the cortical and limbic areas) leads to induction and release of corticortropin-releasing hormone $(\mathrm{CRH})$, which then acts on the pituitary to stimulate the release of corticotropin, which then acts on the adrenal cortex to cause the release of GC (Chrousos 1995). Once GC are released to the circulation they reach a variety of target tissues where, through binding to the GCR, they lead to inhibition of 
AP-1- and NF- $\kappa \mathrm{B}-$ mediated gene induction. One of the most potent activators of the HPA is the pro-inflammatory cytokine IL-1, which can cross the blood-brain barrier and enter the central nervous system (CNS) (Besedovsky et al. 1986). Importantly, the synthesis of IL-1 and other proinflammatory cytokines is controlled by AP-1 and NF- $\mathrm{KB}$ (Barnes \& Karin 1997, Ip \& Davis 1998) and IL-1 itself is a potent activator of JNK and IKB kinase, the protein kinases that lead to activation of AP-1 and NF-KB respectively (Karin 1995, Karin 1999). Thus even a small increase in the level of IL-1 (or TNF) can lead to a much larger increase in IL-1 (or TNF) levels through this feed-forward positive regulatory loop. Once the levels of IL-1 or TNF are increased they can lead to many adverse effects including septic shock. Therefore, it is important to subject the production of IL-1 and other pro-inflammatory cytokines to negative regulation. Such negative regulation is achieved via the HPA axis, which acts as a safety valve to keep the levels of IL-1 and other inflammatory and stress mediators under control.

We found that one of the signaling pathways that activate the HPA axis is none other than the JNK pathway, which also leads to stimulation of AP-1 activity. Using acoustic stimulation as a form of emotional stress we found that JNK1 knockout mice displayed defective induction of c-Jun and c-Fos and a marked reduction in c-Jun N-terminal phosphorylation in certain areas of the CNS, including the hippocampus and hypothalamus (L Chang, S C Dulawa, M A Geyer, C Arias, P E Sawchenko, Y Hu, M E Ellisman, R Johnson \& M Karin, unpublished observations). Thus JNK1, which is a major contributor to total stress-induced JNK activity in different parts of the CNS, is required for up-regulation of AP-1 activity in response to emotional stress. One of the AP-1 target genes whose induction in response to emotional stress is severely attenuated in the JNK1 knockout mice is the CRH gene (L Chang, S C Dulawa, M A Geyer, C Arias, P E Sawchenko, Y Hu, M E Ellisman, R Johnson \& M Karin, unpublished observations). As a result of defective CRH expression, JNK1-deficient mice display an attenuated GC response to stress (L Chang, S C Dulawa, M A Geyer, C Arias, P E Sawchenko, Y Hu, M E Ellisman, R Johnson \& M Karin, unpublished observations). These results provide strong evidence for the involvement of JNK in the central stress circuitry and in activation of the HPA axis.

Thus, as summarized in Fig. 1, JNK activation in the periphery is involved in activation of stress responses, such as the inflammatory response to acute infection. In the CNS, however, the same kinases, especially the JNK1 isoform, lead to activation of the HPA axis, which eventually leads to termination or attenuation of the stress response (Fig. 1). This final step in that important feedback loop is mediated by negative interference between GCR and AP-1, attenuating the production of IL-1 and other inflammatory mediators. Thus the most obvious function of the GCR-AP-1 crosstalk is the maintenance and prevention of homeostasis and presentation of too vigorous a response to stress. In the future it would be interesting to determine the evolutionary origin of this form of gene regulation by GCR and whether it appeared prior to or after the more conventional, but less important, liganddependent gene induction.

\section{Acknowledgements}

L C is supported by NRSA fellowships from the NIH. $\mathrm{M} \mathrm{K}$ is an American Cancer Society Research Professor and work in his laboratory is supported by the NIH and State of California Cancer Research Program.

\section{References}

Alberts AS, Geneste O \& Treisman R 1998 Activation of SRFregulated chromosomal templates by Rho-family GTPases requires a signal that also induces $\mathrm{H} 4$ hyperacetylation. Cell $\mathbf{9 2}$ $475-487$.

Angel P \& Karin M 1991 The role of Jun, Fos and the AP-1 complex in cell-proliferation and transformation. Biochimica et Biophysica Acta 1072 129-157.

Auphan N, DiDonato JA, Rosette C, Helmberg A \& Karin M 1995 Immunosuppression by glucocorticoids: inhibition of NF-kB activity through induction of IkB synthesis. Science 270 286-290.

Barnes PJ \& Karin M 1997 Nuclear factor- $\kappa B$ - A pivotal transcription factor in chronic inflammatory diseases. New England Journal of Medicine 336 1066-1071.

Beato M 1989 Gene regulation by steriod hormones. Cell 56 335344.

Besedovsky H, del Rey A, Sorkin E \& Dinarello CA 1986 Immunoregulatory feedback between interleukin-1 and glucocorticoid hormones. Science 233 652-654.

Braunstein M, Rose AB, Holmes SG, Allis CD \& Broach JR 1993 Transcriptional silencing in yeast is associated with reduced nucleosome acetylation. Genes and Development 7 592-604.

Caelles C, Gonzalez-Sancho JM \& Munoz A 1997 Nuclear hormone receptor antagonism with AP-1 by inhibition of the JNK pathway. Genes and Development 11 3351-3364.

Cavigelli M, Dolfi F, Claret FX \& Karin M 1995 Induction of $c-f o s$ expression through JNK-mediated TCF/Elk-1 phosphorylation. EMBO Journal 14 5957-5964.

Chrousos GP 1995 The hypothalamic-pituitary-adrenal axis and immune-mediated inflammation. New England Journal of Medicine 332 1351-1362.

Cole TJ, Blendy JA, Monaghan AP, Krieglstein K, Schmid W, Aguzzi A, Fantuzzi G, Hummler E, Unsicker K \& Schütz G 1995 Targeted disruption of the glucocorticoid receptor gene blocks adrenergic chromaffin cell development and severely retards lung maturation. Genes and Development 9 1608-1621.

Diamond MA, Miner JN, Yoshinaga SK \& Yamamoto KR 1990 Transcription factor interactions: selectors of positive or negative regulation from a single DNA element. Science 249 1266-1272.

Gonzalez MV, Jimenez B, Berciano MT, Gonzalez-Sancho JM, Caelles C, Lafarga M \& Munoz A 2000 Glucocorticoids antagonize AP-1 by inhibiting the activation/phosphorylation of JNK without affecting its subcellular distribution. Journal of Cell Biology $\mathbf{1 5 0}$ 1199-1208.

Ip YT \& Davis RJ 1998 Signal transduction by the c-Jun NH-2terminal kinase (JNK) - from inflammation to development. Current Opinion in Cell Biology 10 205-219. 
Jonat C, Rahmsdorf HJ, Park KK, Cato ACB, Gebel S, Ponta H \& Herrlich P 1990 Antitumor promotion and anti-inflammation: down-modulation of AP-1 (Fos/Jun) activity by glucocorticoid hormone. Cell 62 1189-1204.

Kallunki T, Deng T, Hibi M \& Karin M. 1996 c-Jun can recruit JNK to phosphorylate dimerization partners via specific docking interactions. Cell 87 929-939.

Kamei Y, Xu L, Heinzel T, Torchia J, Kurokawa R, Gloss B, Lin SC, Heyman RA, Rose DW, Glass CK \& Rosenfeld MG 1996 A $\mathrm{CBP}$ integrator complex mediates transcriptional activation and AP-1 inhibition by nuclear receptors. Cell 85 403-414.

Karin M 1995 The regulation of AP-1 activity by mitogen-activated protein kinases. Journal of Biological Chemistry 270 16483-16486.

Karin M 1998 New twists in gene regulation by glucocorticoid receptor: is DNA binding dispensable? Cell $\mathbf{9 3} 487-490$.

Karin M 1999 The beginning of the end: IKB kinase (IKK) and NF-אB Activation. Journal of Biological Chemistry 274 27339-27342.

Karin M, Haslinger A, Holtgrev H, Richards RI, Krauter P, Westphal H \& Beato M 1984 Characterization of DNA sequences through which cadmium and glucocorticoid hormones induce human metallothionein-IIA gene. Nature 308 513-519.

Kelly CA \& Griffiths ID 1989 Major upper airways obstruction associated with Sjogren's syndrome: a case report and literature review. British Journal of Rheumatology 28 543-545.

Konig H, Ponta H, Rahmsdorf HJ \& Herrlich P 1992 Interference between pathway-specific transcription factors: glucocorticoids antagonize phorbol ester-induced AP-1 activity without altering AP-1 site occupation in vivo. EMBO Journal 11 2241-2246.

Lefstin JA \& Yamamoto KR 1998 Allosteric effects of DNA on transcriptional regulators. Nature 392 885-888.
Reichardt HM, Kaestner KH, Tuckermann J, Kretz O, Wessely O, Bock R, Gass P, Schmid W, Herrlich P, Angel P \& Schutz G 1998 DNA binding of the glucocorticoid receptor is not essential for survival. Cell 93 531-541.

Rogatsky I, Logan SK \& Garabedian MJ 1998 Antagonism of glucocorticoid receptor transcriptional activation by the c-Jun N-terminal kinase. PNAS 95 2050-2055.

Saatcioglu F, Claret FX \& Karin M 1994 Negative transcriptional regulation by nuclear receptors. Seminars in Cancer Biology 266 1719-1723.

Schüle R, Rangarajan P, Kliewer S, Ransone LJ, Bolado J, Yang N, Verma IM \& Evans RM 1990 Functional antagonism between oncoprotein c-Jun and the glucocorticoid receptor. Cell 62 1217-1226.

Smeal T, Binétruy B, Mercola D, Birrer M \& Karin M 1991 Phosphorylation of cJun on serines 63 and 73 is required for oncogenic and transcriptional cooperation with Ha-Ras. Nature 354 494-496.

Swantek JL, Cobb MH \& Geppert TD 1997 Jun N-terminal kinase/stress-activated protein kinase (JNK/SAPK) is required for lipopolysaccharide stimulatonof tumor necrosis factor $\alpha$ (TNF- $\alpha$ ) translation: glucocorticoids inhibit TNF- $\alpha$ translation by blocking JNK/SAPK. Molecular and Cellular Biology 17 6274-6282.

Yang-Yen HF, Chambard JC, Sun YL, Smeal T, Schmidt TJ, Drouin J \& Karin M 1990 Transcriptional interference between cJun and the glucocorticoid receptor: mutual inhibition of DNA-binding due to direct protein-protein interaction. Cell 62 1205-1215.

Received 6 December 2000

Accepted 25 January 2001 